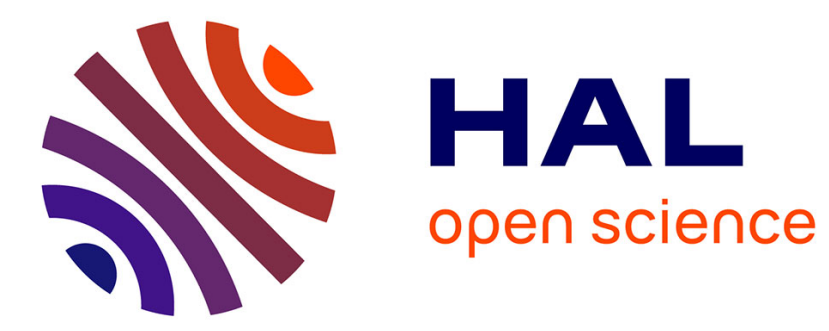

\title{
Filtration Law in Porous Media with Poor Separation of Scales
}

\author{
Jean-Louis Auriault, Christian Geindreau, Claude Boutin
}

\section{To cite this version:}

Jean-Louis Auriault, Christian Geindreau, Claude Boutin. Filtration Law in Porous Media with Poor Separation of Scales. Transport in Porous Media, 2005, 60 (1), pp.89-108. 10.1007/s11242-004-36497. hal-00943739

\section{HAL Id: hal-00943739 \\ https://hal.science/hal-00943739}

Submitted on 8 Feb 2014

HAL is a multi-disciplinary open access archive for the deposit and dissemination of scientific research documents, whether they are published or not. The documents may come from teaching and research institutions in France or abroad, or from public or private research centers.
L'archive ouverte pluridisciplinaire HAL, est destinée au dépôt et à la diffusion de documents scientifiques de niveau recherche, publiés ou non, émanant des établissements d'enseignement et de recherche français ou étrangers, des laboratoires publics ou privés. 


\title{
Filtration Law in Porous Media with Poor Separation of Scales
}

\author{
JEAN-LOUIS AURIAULT ${ }^{1, *}$, CHRISTIAN GEINDREAU ${ }^{1}$ and \\ CLAUDE BOUTIN ${ }^{2}$ \\ ${ }^{1}$ Laboratoire Sols Solides Structures (3S), UJF, INPG, CNRS, Domaine Universitaire, BP \\ 53, 38041 Grenoble Cedex, France \\ ${ }^{2}$ Laboratoire Géomatériaux, DGCB CNRS URA 1652, Ecole Nationale des Travaux \\ publiques de l'Etat, rue Maurice Audin, 69518 Vaulx en Velin, France
}

\begin{abstract}
We investigate the validity of Darcy's law when the separation of scales is poor. We use the method of multi-scale asymptotic expansions which gives the macroscopic behaviour from the pore scale description. The first order approximation is the Darcy's law. When the separation of scales is poor, eventual correctors to Darcy's law are obtained by investigating the following orders of approximation, thus enabling us to study its robustness. We investigate the two first correctors. Thus, the accuracy of the macroscopic flow law is improved from $\mathcal{O}(\varepsilon)$ to $\mathcal{O}\left(\varepsilon^{3}\right)$, where $\varepsilon$ is the separation of scale parameter. The second corrector shows a Brinkman's term. For macroscopically homogeneous porous media, the first corrector cancels out, that points out the robustness of Darcy's law in this case.
\end{abstract}

Key words: porous media, flow law, poor separation of scales, Brinkman's law homogenization.

\section{Introduction}

It is well admitted that the existence of continuum behaviours that are macroscopically equivalent to finely heterogeneous media needs a "good" separation of scales. If $l$ and $L$ are the characteristic lengths at the local and the macroscopic scale, respectively, their ratio should obey

$$
\varepsilon=\frac{l}{L} \ll 1
$$

The above condition is required, in particular, for flow in rigid porous media to obtain the macroscopic flow law, i.e. the Darcy's law (Ene and Sanchez-Palencia, 1975; Sanchez-Palencia, 1980).

\footnotetext{
*Author for correspondence: e-mail: jean-louis.auriault@hmg.ing.fr
} 
The aim of this paper is to investigate the filtration law in rigid porous matrices for steady-state slow flow of an incompressible viscous Newtonian fluid when the separation of scales is poor

$$
\varepsilon=\frac{l}{L}<1 \text {. }
$$

That can be encountered in two typical situations. The first one occurs when the porous medium is macroscopically heterogeneous, when the macroscopic characteristic length $L$ associated to the macroscopic heterogeneities is not "very" large compared to the characteristic length $l$ of the pores. For such media, length $L$ can be estimated by $L \approx K /|\nabla K|$, where $K$ is the permeability. When the macroscopic gradient of the permeability $\nabla K$ is large, the ratio $l / L$ may be not "very" small and the separation of scales is poor.

The second typical situation corresponds to large gradients of pressure which are applied to macroscopically homogeneous media, such as those encountered near wells. The macroscopic characteristic length $L \approx p /|\nabla p|$ associated to this gradient of pressure could be not "very" large compared to $l$. Again, the separation of scale ratio is not "very" small. The question under consideration is: how must the Darcy's law be modified when the separation of scales is poor?

To our knowledge, cases of poor separation of scales have received little attention in the literature. However, an important work is presented by Goyeau et al. (1997, 1999). The authors investigate the permeability in a dendritic mushy zone, which is generally a nonhomogeneous porous structure. They make use of the volume averaging method to obtain corrector terms to Darcy's law. As recognized by the authors, the full solution is still out of reach. Even when the separation of scales is acceptable, some investigations need a corrector to Darcy's law: see, e.g., the study of dispersion in porous media (Auriault and Adler, 1995).

In the present paper, we use an upscaling technique, i.e. the method of multiple scale expansions to determine the macroscopic flow from its description at the pore scale. Heterogeneous systems, as for example porous media, may enable us to investigate an equivalent macroscopic continuous system if the condition of separation of scales (1) is verified (Bensoussan et al., 1978; Sanchez-Palencia, 1980). The cases of poor separation of scales (2) will be investigated by introducing correctors to the case of good separation of scales. The method in use enables us investigating all possible situations of poor separation of scales.

The macroscopic equivalent model is obtained from the description at the heterogeneity scale by Auriault (1991): (i) assuming the medium to be locally periodic, without loss of generality: consider a right-angled parallelepipedic representative elementary volume (REV) of a random porous 
medium. Complete this REV by three plane symmetries with respect to three of its perpendicular faces. Both the so obtained periodic medium and the random medium obey similar macroscopic behaviours, with a possibly modified anisotropy. For an investigation of random porous media without the periodicity condition, see Murdoch and Hassanizadeh (2002). Nevertheless, the medium can be heterogeneous at the macroscopic scale; (ii) writing the local description in a dimensionless form; (iii) evaluating the dimensionless numbers with respect to the scale ratio $\varepsilon$; (iv) looking for the unknown fields in the form of asymptotic expansions in powers of $\varepsilon$; (v) solving the successive boundary-value problems that are obtained after introducing these expansions in the local dimensionless description. The macroscopic equivalent model is obtained from compatibility conditions which are the necessary conditions for the existence of solutions to the boundary-value problems. When considering the slow stationary flow of a Newtonian fluid, the method yields at the first order of approximation the well known Darcy's law.

In the present paper, we consider a poor separation of scales, i.e. when the parameter $\varepsilon$ is not very small. In such a case, Darcy's law becomes a poor approximation for describing the flow, with a relative error $\mathcal{O}(\varepsilon)$. The method consists in looking for correctors to Darcy's law. In the paper we consider the two first correctors, which yield a modified flow law with a relative error $\mathcal{O}\left(\varepsilon^{3}\right)$. The porous medium is described and the pore scale behaviour is given in part 2. The upscaling is performed in part 3, which gives at the first order of approximation, i.e. the Darcy's law. First and second correctors are investigated. The second corrector shows a Brinkman term. For macroscopically homogeneous porous media and large gradients of pressure, the first corrector cancels out that points out the robustness of Darcy's law in this case. Finally, part 4 is devoted to an analytical example.

\section{Local Flow Description and Estimations}

Consider the flow of an incompressible Newtonian liquid through a porous medium. The porous medium is spatially strictly or locally periodic and consists of repeated unit cells (parallelepipeds), see Figure 1. There are two characteristic length scales in this problem: the characteristic microscopic length scale $l$ of the pores and of the unit cell (we assume no separation of scales between these two characteristic lengths), and the macroscopic length scale that may be represented by either the macroscopic pressure drop scale or by the sample size scale. For simplicity, we assume both macroscopic length scales to be of similar order of magnitude, $\mathcal{O}(L)$. Moreover, we assume that the two length scales $l$ and $L$ are separated

$l \ll L$ 


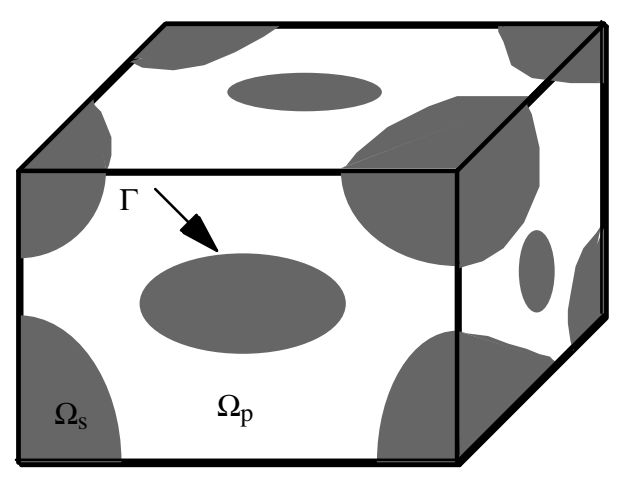

Figure 1. Schematic view of a period of the porous medium.

The unit cell is denoted by $\Omega$ and is bounded by $\partial \Omega$, the fluid part of the unit cell is denoted by $\Omega_{p}$, and the fluid-solid interface inside the unit cell is $\Gamma$. Relatively to the porous matrix frame, the momentum balance for the incompressible viscous Newtonian liquid is

$$
\mu \frac{\partial^{2} v_{i}}{\partial X_{j} \partial X_{j}}-\frac{\partial p}{\partial X_{i}}=0 \text { in } \Omega_{p},
$$

where $\mathbf{v}$ is the velocity vector relative to the matrix frame, $p$ is the pressure and $\mu$ is the viscosity assumed as a constant. Gravitational acceleration is included in the pressure term. Equation (4) is completed by the incompressibility condition and the adherence condition on $\Gamma$

$$
\begin{aligned}
\frac{\partial v_{i}}{\partial X_{i}} & =0 & & \text { in } \Omega_{p}, \\
v_{i} & =0 & & \text { on } \Gamma .
\end{aligned}
$$

The ratio between microscopic and macroscopic length scales is small (but not too small). The fundamental perturbation parameter $\varepsilon$ is chosen to be

$$
\varepsilon=\frac{l}{L}, \quad \varepsilon \ll 1
$$

To render dimensionless the system (4-6), we use the local length scale of a pore $l$ as the characteristic length scale for the variations of the differential operators: we apply the so-called microscopic point of view (Auriault, 1991). Therefore, we introduce the small scale dimensionless space variable $\mathbf{y}=\mathbf{X} / l$. Since scales are separated, a second dimensionless space variable is defined, $\mathbf{x}=\mathbf{X} / L=\varepsilon \mathbf{y}$, which is well suited to describing the macroscopic variations. Other characteristic quantities are denoted by the subscript $c$, whereas dimensionless quantities are shown by an asterisk. When the porous medium is locally periodic, the period depends on 
the space variable $\mathbf{x}$. Then, the system (4-6) defines a single dimensionless number $\mathcal{Q}$ which is the ratio of pressure to viscous forces. We obtain

$$
\mathcal{Q}=\frac{p_{c} l}{\mu_{c} v_{c}}
$$

The estimate for $\mathcal{Q}$ comes from a phenomenological argument, i.e. the viscous flow is locally driven by a macroscopic pressure gradient

$$
\frac{\mu_{c} v_{c}}{l^{2}}=\mathcal{O}\left(\frac{p_{c}}{L}\right)
$$

and thus

$$
\mathcal{Q}=\mathcal{O}\left(\varepsilon^{-1}\right)
$$

The dimensionless set that describes the flow is in the form

$$
\begin{aligned}
& \mu^{*} \frac{\partial^{2} v_{i}^{*}}{\partial y_{j} \partial y_{j}}-\varepsilon^{-1} \frac{\partial p^{*}}{\partial y_{i}}=0, \\
& \frac{\partial v_{i}^{*}}{\partial y_{i}}=0 \text { in } \Omega_{p}^{*}, \\
& v_{i}^{*}=0 \text { on } \Gamma^{*} .
\end{aligned}
$$

\section{Homogenization}

Following the multiple scale expansion technique (Bensoussan et al., 1978; Sanchez-Palencia, 1980), the velocity $\mathbf{v}^{*}$ and the pressure fluctuation $p^{*}$ are looked for in the form of asymptotic expansions of powers of $\varepsilon$

$$
\begin{aligned}
\mathbf{v}^{*} & =\mathbf{v}^{* 0}(\mathbf{x}, \mathbf{y})+\varepsilon \mathbf{v}^{* 1}(\mathbf{x}, \mathbf{y})+\varepsilon^{2} \mathbf{v}^{* 2}(\mathbf{x}, \mathbf{y})+\cdots, \\
p & =p^{* 0}(\mathbf{x}, \mathbf{y})+\varepsilon p^{* 1}(\mathbf{x}, \mathbf{y})+\varepsilon^{2} p^{* 2}(\mathbf{x}, \mathbf{y})+\cdots,
\end{aligned}
$$

where the different terms of the asymptotic expansions are $\Omega^{*}$-periodic with respect to variable $\mathbf{y}$. Substituting these expansions in the set (11-13) gives, by identification of equal power of $\varepsilon$, successive boundary value problems to be investigated. The lowest order approximation of the pressure requires that

$$
\frac{\partial p^{* 0}}{\partial y_{i}}=0, \quad p^{* 0}=p^{* 0}(\mathbf{x}) .
$$




\subsection{DARCY'S LAW}

We first recall the first order macroscopic flow description (SanchezPalencia, 1980). The first order approximation $\mathbf{v}^{* 0}$ of the velocity and the second order approximation $p^{* 1}$ of the pressure are determined by the following set

$$
\begin{aligned}
& \mu^{*} \frac{\partial^{2} v_{i}^{* 0}}{\partial y_{j} \partial y_{j}}-\frac{\partial p^{* 0}}{\partial x_{i}}-\frac{\partial p^{* 1}}{\partial y_{i}}=0, \\
& \frac{\partial v_{i}^{* 0}}{\partial y_{i}}=0 \text { in } \Omega_{p}^{*}, \\
& v_{i}^{* 0}=0 \text { on } \Gamma^{*},
\end{aligned}
$$

where $\mathbf{v}^{* 0}$ and $p^{* 1}$ are $\Omega^{*}$-periodic. The above system has a unique solution $\mathbf{v}^{* 0}$ which is a linear vector function of $\partial p^{* 0} / \partial x_{i}$ (Ene and SanchezPalencia, 1975; Sanchez-Palencia, 1980)

$$
v_{i}^{* 0}=-\frac{k_{i j}^{*}(\mathbf{x}, \mathbf{y})}{\mu^{*}} \frac{\partial p^{* 0}}{\partial x_{j}},
$$

where the tensor field $\mathbf{k}^{*}$ depends on $\mathbf{y}$, and possibly on $\mathbf{x}$ when the porous medium is macroscopically heterogeneous. From Equation (17), $p^{* 1}$ can be put in the form

$$
p^{* 1}=-a_{j}^{*}(\mathbf{x}, \mathbf{y}) \frac{\partial p^{* 0}}{\partial x_{j}}+\bar{p}^{* 1}(\mathbf{x}), \quad\left\langle a_{i}^{*}\right\rangle_{\Omega_{p}^{*}}=\frac{1}{\Omega_{p}^{*}} \int_{\Omega_{p}^{*}} a_{j}^{*} \mathrm{dV}^{*}=0 .
$$

Finally, the volume balance (12) gives at the second order

$$
\frac{\partial v_{i}^{* 1}}{\partial y_{i}}+\frac{\partial v_{i}^{* 0}}{\partial x_{i}}=0 \text { in } \Omega_{p}^{*} \text {. }
$$

By integrating over $\Omega_{p}^{*}$, we obtain

$$
\frac{\partial\left\langle v_{i}^{* 0}\right\rangle}{\partial x_{i}}=0, \quad\left\langle v_{i}^{* 0}\right\rangle=-\frac{K_{i j}^{*}(\mathbf{x})}{\mu^{*}} \frac{\partial p^{* 0}}{\partial x_{j}}, \quad K_{i j}^{*}=\frac{1}{\Omega^{*}} \int_{\Omega_{p}^{*}} k_{i j}^{*} \mathrm{dV}^{*} .
$$

Returning to dimensional quantities yields

$$
\frac{\partial\left\langle v_{i}^{0}\right\rangle}{\partial X_{i}}=0, \quad\left\langle v_{i}^{0}\right\rangle=-\frac{K_{i j}^{e f f}(\mathbf{X})}{\mu} \frac{\partial p^{0}}{\partial X_{j}}, \quad K_{i j}^{e f f}=l^{2} K_{i j}^{*},
$$

which gives the first order approximation description of the fluid flow

$$
\frac{\partial\left\langle v_{i}\right\rangle}{\partial X_{i}}=\mathcal{O}\left(\varepsilon \frac{\partial\left\langle v_{i}\right\rangle}{\partial X_{i}}\right), \quad\left\langle v_{i}\right\rangle=-\frac{K_{i j}^{e f f}(\mathbf{X})}{\mu} \frac{\partial p}{\partial X_{j}}+\mathcal{O}\left(\varepsilon\left\langle v_{i}\right\rangle\right) .
$$


The second relation in (25) stands for the Darcy's law. It is possible to show, (Ene and Sanchez-Palencia, 1975; Sanchez-Palencia, 1980), that the permeability $\mathbf{K}^{e f f}$ is a symmetrical tensor

$$
K_{i j}^{e f f}=K_{j i}^{e f f} .
$$

When $\varepsilon$ is not small, Equation (25) gives a poor approximation of the behaviour. A better behaviour is obtained by introducing a corrector.

\subsection{FIRST CORRECTOR}

The second order approximation $\mathbf{v}^{* 1}$ of the velocity and the third order approximation $p^{* 2}$ of the pressure are determined by the following set

$$
\begin{aligned}
& \mu^{*} \frac{\partial^{2} v_{i}^{* 1}}{\partial y_{j} \partial y_{j}}+2 \mu^{*} \frac{\partial^{2} v_{i}^{* 0}}{\partial y_{j} \partial x_{j}}-\frac{\partial p^{* 1}}{\partial x_{i}}-\frac{\partial p^{* 2}}{\partial y_{i}}=0, \\
& \frac{\partial v_{i}^{* 1}}{\partial y_{i}}+\frac{\partial v_{i}^{* 0}}{\partial x_{i}}=0 \text { in } \Omega_{p}^{*}, \\
& v_{i}^{* 1}=0 \text { on } \Gamma^{*},
\end{aligned}
$$

where $\mathbf{v}^{* 1}$ and $p^{* 2}$ are $\Omega^{*}$-periodic. By using the compatibility condition (23), balance (27) becomes

$$
\frac{\partial v_{i}^{* 1}}{\partial y_{i}}+\frac{\partial\left(v_{i}^{* 0}-\phi^{-1}\left\langle v_{i}^{* 0}\right\rangle\right)}{\partial x_{i}}=0 \text { in } \Omega_{p}^{*},
$$

where $\phi$ is the porosity. Introducing now the expressions (20) and (21) for $\mathbf{v}^{* 0}$ and $p^{* 1}$, respectively, changes (26) and (29) into

$$
\begin{aligned}
& \mu^{*} \frac{\partial^{2} v_{i}^{* 1}}{\partial y_{j} \partial y_{j}}-\frac{\partial p^{* 2}}{\partial y_{i}}=\left(2 \frac{\partial k_{i k}^{*}}{\partial y_{j}}-a_{k}^{*} I_{i j}\right) \frac{\partial^{2} p^{* 0}}{\partial x_{j} \partial x_{k}} \\
&+\left(2 \frac{\partial^{2} k_{i k}^{*}}{\partial y_{j} \partial x_{j}}-\frac{\partial a_{k}^{*}}{\partial x_{i}}\right) \frac{\partial p^{* 0}}{\partial x_{k}}+\frac{\partial \bar{p}^{* 1}}{\partial x_{i}}, \\
& \frac{\partial v_{i}^{* 1}}{\partial y_{i}}-\frac{1}{\mu^{*}} \frac{\partial\left(k_{i j}^{*}-\phi^{-1} K_{i j}^{*}\right)}{\partial x_{i}} \frac{\partial p^{* 0}}{\partial x_{j}}-\frac{1}{\mu^{*}}\left(k_{i j}^{*}-\phi^{-1} K_{i j}^{*}\right) \frac{\partial^{2} p^{* 0}}{\partial x_{i} \partial x_{j}}=0 \text { in } \Omega_{p}^{*} .
\end{aligned}
$$

The system (28-30-31) verifies the compatibility condition. The solution is in the form

$$
\begin{aligned}
& v_{i}^{* 1}=-\frac{k_{i j}^{*}}{\mu^{*}} \frac{\partial \bar{p}^{* 1}}{\partial x_{j}}-\frac{l_{i j}^{*}}{\mu^{*}} \frac{\partial p^{* 0}}{\partial x_{j}}-\frac{n_{i j k}^{*}}{\mu^{*}} \frac{\partial^{2} p^{* 0}}{\partial x_{j} \partial x_{k}}, \\
& p^{* 2}=-a_{j}^{*} \frac{\partial \bar{p}^{* 1}}{\partial x_{j}}-b_{j}^{*} \frac{\partial p^{* 0}}{\partial x_{j}}-d_{j k}^{*} \frac{\partial^{2} p^{* 0}}{\partial x_{j} \partial x_{k}}+\bar{p}^{* 2}(\mathbf{x}),
\end{aligned}
$$


where tensor $\mathbf{k}^{*}$ and vector $\mathbf{a}^{*}$ are defined above and $\mathbf{l}^{*}, \mathbf{n}^{*}, \mathbf{b}^{*}$ with $\left\langle\mathbf{b}^{*}\right\rangle=0$ and $\mathbf{d}^{*}$ with $\left\langle\mathbf{d}^{*}\right\rangle=0$ are tensorial functions of $\mathbf{x}$ and $\mathbf{y}$. When the porous medium is macroscopically homogeneous, $l_{i j}^{*}=0$ and $b_{j}^{*}=0$.

The volume balance (12) gives at the third order

$$
\frac{\partial v_{i}^{* 2}}{\partial y_{i}}+\frac{\partial v_{i}^{* 1}}{\partial x_{i}}=0 \text { in } \Omega_{p}^{*} \text {. }
$$

By integrating over $\Omega_{p}^{*}$, we obtain

$$
\begin{aligned}
& \frac{\partial\left\langle v_{i}^{* 1}\right\rangle}{\partial x_{i}}=0, \\
& \left\langle v_{i}^{* 1}\right\rangle=-\frac{K_{i j}^{*}(\mathbf{x})}{\mu^{*}} \frac{\partial \bar{p}^{* 1}}{\partial x_{j}}-\frac{L_{i j}^{*}(\mathbf{x})}{\mu^{*}} \frac{\partial p^{* 0}}{\partial x_{j}}-\frac{N_{i j k}^{*}(\mathbf{x})}{\mu^{*}} \frac{\partial^{2} p^{* 0}}{\partial x_{j} \partial x_{k}}, \\
& L_{i j}^{*}=\left\langle l_{i j}^{*}\right\rangle, \quad N_{i j k}^{*}=\left\langle n_{i j k}^{*}\right\rangle .
\end{aligned}
$$

Tensor $\mathbf{N}^{*}$ can be calculated from tensor fields $\mathbf{k}^{*}$ and $\mathbf{a}^{*}$ without solving the boundary value problem (28-30-31), see relation (82) in Appendix A. In dimensional form, we obtain

$$
\begin{aligned}
& \frac{\partial\left\langle v_{i}^{1}\right\rangle}{\partial X_{i}}=0, \\
& \left\langle v_{i}^{1}\right\rangle=-\frac{K_{i j}(\mathbf{X})}{\mu} \frac{\partial \bar{p}^{1}}{\partial X_{j}}-\frac{L_{i j}(\mathbf{X})}{\mu} \frac{\partial p^{0}}{\partial X_{j}}-\frac{N_{i j k}(\mathbf{X})}{\mu} \frac{\partial^{2} p^{0}}{\partial X_{j} \partial X_{k}}, \\
& L_{i j}=l^{2} L_{i j}^{*}, \quad N_{i j k}=l^{2} L N_{i j k}^{*}
\end{aligned}
$$

\subsection{FIRST CORRECTED MACROSCOPIC BEHAVIOUR}

Let us introduce corrected macroscopic velocity and pressure in the form

$$
\begin{aligned}
\left\langle v_{i}\right\rangle & =\left\langle v_{i}^{0}\right\rangle+\varepsilon\left\langle v_{i}^{1}\right\rangle+\mathcal{O}\left(\varepsilon^{2}\left\langle v_{i}\right\rangle\right), \\
\langle p\rangle & =p^{0}+\varepsilon\left\langle p^{1}\right\rangle+\mathcal{O}\left(\varepsilon^{2}\langle p\rangle\right) .
\end{aligned}
$$

By adding term by term Equation $\left(24_{2}\right)$ and Equation (38) multiplied by $\varepsilon$, we obtain a corrected Darcy's law in the form

$$
\left\langle v_{i}\right\rangle=-\frac{1}{\mu}\left(\left(K_{i j}^{e f f}+L_{i j}^{e f f}\right) \frac{\partial p}{\partial X_{j}}-\frac{N_{i j k}^{e f f}}{\mu} \frac{\partial^{2} p}{\partial X_{j} \partial X_{k}}+\mathcal{O}\left(\varepsilon^{2}\left\langle v_{i}\right\rangle\right)\right),
$$

with

$$
\frac{\partial\left\langle v_{i}\right\rangle}{\partial X_{i}}=\mathcal{O}\left(\varepsilon^{2} \frac{\partial\left\langle v_{i}\right\rangle}{\partial X_{i}}\right) .
$$


The corrected macroscopic behaviour is now within a relative error $\mathcal{O}\left(\varepsilon^{2}\right)$. The effective parameters verify

$$
\mathbf{K}^{e f f}=\mathcal{O}\left(l^{2}\right), \quad \mathbf{L}^{e f f}=\varepsilon \mathbf{L}=\mathcal{O}\left(\frac{l^{3}}{L}\right), \quad \mathbf{N}^{e f f}=\varepsilon \mathbf{N}=\mathcal{O}\left(l^{3}\right)
$$

By construction tensor $\mathbf{N}$ is symmetrical about its last two indices. By following a similar route as for the permeability $\mathbf{K}$, it is possible to show its antisymmetry about its two first indices (see the Appendix A)

$$
N_{i j k}^{e f f}=N_{i k j}^{e f f}, \quad N_{i j k}^{e f f}=-N_{j i k}^{e f f} .
$$

It is remarkable to note that for a flow through a macroscopically homogeneous porous medium, we have $L_{i j}^{\text {eff }}=0$, and, due to the properties of tensor $\mathbf{N}^{e f f}$, Equation (40) then reduces to

$$
\frac{\partial}{\partial X_{i}}\left(\frac{K_{i j}^{e f f}}{\mu} \frac{\partial p}{\partial X_{j}}\right)=\mathcal{O}\left(\varepsilon^{2} \frac{\partial\left\langle v_{i}\right\rangle}{\partial X_{i}}\right),
$$

which is similar to the first order approximation (23), but with a relative error $\mathcal{O}\left(\varepsilon^{2}\right)$. That shows the robustness of Darcy's law in this case. When the porous medium is macroscopically heterogeneous, tensor $\mathbf{L}^{\text {eff }}$ does not generally cancel out. In such cases, the correction to Darcy's law is $\mathcal{O}(\varepsilon)$.

\subsection{PROPERTIES OF THE FLOW LAW}

Let us first investigate the physical meaning of the volume averaged velocity $\langle\mathbf{v}\rangle$. Consider the identity

$$
\frac{\partial}{\partial y_{i}}\left(y_{j} v_{i}\right) \equiv y_{j} \frac{\partial v_{i}}{\partial y_{i}}+v_{j}
$$

Let $v_{i}=v_{i}^{* 0}$. By using the dimensional form of (18) and integrating (42) on $\Omega_{p}^{*}$, we obtain after some transformation

$$
\left\langle v_{i}^{* 0}\right\rangle=\frac{1}{\Sigma_{i}^{*}} \int_{\Sigma_{p i}^{*}} v_{i}^{* 0} \mathrm{dV}^{*},
$$

where the right hand member represents a surface average, i.e., a flux. The surface $\Sigma_{i}^{*}$ is the cross-section of the period perpendicular to axis $\mathbf{e}_{i}$ and $\Sigma_{p i}^{*}$ its part in $\Omega_{p}^{*}$. Therefore, $\left\langle\mathbf{v}^{* 0}\right\rangle=\left\langle\mathbf{v}^{* 0}\right\rangle_{\Sigma^{*}}$ is a Darcy's velocity. Let now $v_{i}=v_{i}^{* 1}$. On the contrary to $\mathbf{v}^{* 0}$, velocity $\mathbf{v}^{* 1}$ is not divergence free, see (27). Therefore its volume average is not a flux and we obtain for the corrected Darcy's flux 


$$
\begin{aligned}
& \left\langle v_{i}\right\rangle_{\Sigma}=-\frac{1}{\mu}\left(K_{i j}^{e f f}+L_{i j}^{e f f}-L_{i j}^{1 e f f}\right) \frac{\partial p}{\partial X_{j}} \\
& -\frac{1}{\mu}\left(N_{i j k}^{e f f}-N_{i j k}^{1 e f f}\right) \frac{\partial^{2} p}{\partial X_{j} \partial X_{k}}+\mathcal{O}\left(\varepsilon^{2}\left\langle v_{i}\right\rangle_{\Sigma}\right),
\end{aligned}
$$

with

$$
L_{i j}^{1 e f f}=l^{2}\left\langle y_{i} \frac{\partial k_{k j}^{*}}{\partial x_{k}}\right\rangle, \quad N_{i j k}^{1 e f f}=l^{3}\left\langle y_{i} k_{k j}^{*}\right\rangle .
$$

In case of macroscopic isotropy, third order tensors are scalar multiple of the permutation tensor. Since by construction, $\mathbf{N}^{\text {eff }}$ and $\mathbf{N}^{\text {leff }}$ are symmetrical with respect to their two last indices, they cancel out.

\subsection{SECOND CORRECTOR}

In some cases, the second corrector of the velocity may be needed. The third order approximation of the velocity $\mathbf{v}^{* 2}$ and the forth order approximation of the pressure $p^{* 3}$ are determined by the following set

$$
\begin{aligned}
& \mu^{*} \frac{\partial^{2} v_{i}^{* 2}}{\partial y_{j} \partial y_{j}}+\mu^{*} \frac{\partial^{2} v_{i}^{* 0}}{\partial x_{j} \partial x_{j}}+2 \mu^{*} \frac{\partial^{2} v_{i}^{* 1}}{\partial y_{j} \partial x_{j}}-\frac{\partial p^{* 2}}{\partial x_{i}}-\frac{\partial p^{* 3}}{\partial y_{i}}=0 \text { in } \Omega_{p}^{*}, \\
& \frac{\partial v_{i}^{* 2}}{\partial y_{i}}+\frac{\partial v_{i}^{* 1}}{\partial x_{i}}=0 \text { in } \Omega_{p}^{*}, \\
& v_{i}^{* 2}=0 \text { on } \Gamma^{*},
\end{aligned}
$$

where $\mathbf{v}^{* 2}$ and $p^{* 3}$ are $\Omega$-periodic. By subtracting the compatibility condition (35), Equation (46) is changed into

$$
\frac{\partial v_{i}^{* 2}}{\partial y_{i}}+\frac{\partial\left(v_{i}^{* 1}-\phi^{-1}\left\langle v_{i}^{* 1}\right\rangle\right)}{\partial x_{i}}=0 \text {. }
$$

The above set now verifies the compatibility condition, that ensures the existence of a solution. Introducing the expressions (20), (32), (21) and (33) changes (45), (47) and (48) into a set which solution $\mathbf{v}^{* 2}$ and $p^{* 3}$ can be put in the form

$$
\begin{aligned}
v_{i}^{* 2}= & -\frac{k_{i j}^{*}}{\mu^{*}} \frac{\partial \bar{p}^{* 2}}{\partial x_{j}}-\frac{l_{i j}^{*}}{\mu^{*}} \frac{\partial \bar{p}^{* 1}}{\partial x_{j}}-\frac{m_{i j}^{*}}{\mu^{*}} \frac{\partial p^{* 0}}{\partial x_{j}} \\
& -\frac{n_{i j k}^{*}}{\mu^{*}} \frac{\partial^{2} \bar{p}^{* 1}}{\partial x_{j} \partial x_{k}}-\frac{o_{i j k}^{*}}{\mu^{*}} \frac{\partial^{2} p^{* 0}}{\partial x_{j} \partial x_{k}}-\frac{p_{i j k l}^{*}}{\mu^{*}} \frac{\partial^{3} p^{* 0}}{\partial x_{j} \partial x_{k} \partial x_{k}}, \\
p_{i}^{* 3}= & -a_{i}^{*} \frac{\partial \bar{p}^{* 2}}{\partial x_{i}}-b_{i}^{*} \frac{\partial \bar{p}^{* 1}}{\partial x_{i}}-c_{i}^{*} \frac{\partial p^{* 0}}{\partial x_{i}} \\
& -d_{i j}^{*} \frac{\partial^{2} \bar{p}^{* 1}}{\partial x_{i} \partial x_{j}}-e_{i j}^{*} \frac{\partial^{2} p^{* 0}}{\partial x_{i} \partial x_{j}}-f_{i j k}^{*} \frac{\partial^{3} p^{* 0}}{\partial x_{i} \partial x_{j} \partial x_{k}},
\end{aligned}
$$


where $\mathbf{m}^{*}, \mathbf{o}^{*}, \mathbf{p}^{*}, \mathbf{c}^{*}, \mathbf{e}^{*}$ and $\mathbf{f}^{*}$ with $\left\langle\mathbf{c}^{*}\right\rangle=\left\langle\mathbf{e}^{*}\right\rangle=\left\langle\mathbf{f}^{*}\right\rangle=0$ are new tensorial functions of $\mathbf{x}$ and $\mathbf{y}$. The volume balance (12) gives at the forth order

$$
\frac{\partial v_{i}^{* 3}}{\partial y_{i}}+\frac{\partial v_{i}^{* 2}}{\partial x_{i}}=0 \text { in } \Omega_{p}^{*} .
$$

By integrating over $\Omega_{p}^{*}$, we obtain

$$
\begin{aligned}
\frac{\partial\left\langle v_{i}^{* 2}\right\rangle}{\partial x_{i}}= & 0, \\
\left\langle v_{i}^{* 2}\right\rangle= & -\frac{K_{i j}^{*}(\mathbf{x})}{\mu^{*}} \frac{\partial \bar{p}^{* 2}}{\partial x_{j}}-\frac{L_{i j}^{*}(\mathbf{x})}{\mu^{*}} \frac{\partial \bar{p}^{* 1}}{\partial x_{j}}-\frac{M_{i j}^{*}(\mathbf{x})}{\mu^{*}} \frac{\partial p^{* 0}}{\partial x_{j}} \\
& -\frac{N_{i j k}^{*}}{\mu^{*}} \frac{\partial^{2} \bar{p}^{* 1}}{\partial x_{j} \partial x_{k}}-\frac{O_{i j k}^{*}}{\mu^{*}} \frac{\partial^{2} p^{* 0}}{\partial x_{j} \partial x_{k}}-\frac{P_{i j k l}^{*}}{\mu^{*}} \frac{\partial^{3} p^{* 0}}{\partial x_{j} \partial x_{k} \partial x_{l}} .
\end{aligned}
$$

As shown below the third derivative term in (53) corresponds to a Brinkman's term. Tensor $\mathbf{P}^{*}$ can be calculated from tensor fields $\mathbf{k}^{*}, \mathbf{a}^{*}, \mathbf{n}^{*}$ and $\mathbf{d}^{*}$, without solving the boundary value problem (45-47-48), see relation (89) in Appendix B. The dimensional form is easily obtained by following the same route as in the previous subsection. When the medium is isotropic, third order tensors $\mathbf{O}^{*}$ and $\mathbf{N}^{*}$ cancels out. When the medium is macroscopically homogeneous, $\mathbf{L}^{*}$ and $\mathbf{M}^{*}$ cancels out too. The third derivative term in (53) is a Brinkman's term. To see this, consider a macroscopically homogeneous isotropic porous medium. To the second order of approximation, the macroscopic behaviour reduces to

$$
\left\langle v_{i}\right\rangle=-\frac{K^{e f f}}{\mu} \frac{\partial p}{\partial X_{i}}-\frac{P^{e f f}}{\mu} \frac{\partial^{3} p}{\partial X_{i} \partial X_{k} \partial X_{k}}+\mathcal{O}\left(\varepsilon^{3}\left\langle v_{i}\right\rangle\right)
$$

with

$$
\frac{\partial\left\langle v_{i}\right\rangle}{\partial X_{i}}=\mathcal{O}\left(\varepsilon^{3} \frac{\partial\left\langle v_{i}\right\rangle}{\partial X_{i}}\right)
$$

where $K^{e f f}$ and $P^{e f f}$ are scalars and

$$
K^{e f f}=\mathcal{O}\left(l^{2}\right), \quad P^{e f f}=\mathcal{O}\left(l^{4}\right) .
$$

After remembering that the third derivative term in (54) is much smaller than the first derivative term, flow law (54) can be put in the form

$$
\left\langle v_{i}\right\rangle=-\frac{K^{e f f}}{\mu} \frac{\partial p}{\partial X_{j}}+\frac{P^{e f f}}{K^{e f f}} \frac{\partial^{2}\left\langle v_{i}\right\rangle}{\partial X_{j} \partial X_{j}}+\mathcal{O}\left(\varepsilon^{3}\left\langle v_{i}\right\rangle\right),
$$


which shows a Brinkman's correction term proportional to the Laplacian of the velocity. This term is of relative weight $\mathcal{O}\left(\varepsilon^{2}\right)$.

\section{Analytical Example}

We investigate a macroscopically heterogeneous porous medium which the pore system consists of "locally parallel" plane fissures with slowly varying thickness $h\left(X_{1}\right): h^{*}=h^{*}\left(x_{1}\right)$. Median plane of the considered fissure is $\left(\mathbf{e}_{1}, \mathbf{e}_{2}\right)$ and the medium is periodic of constant period $l$ in the $\mathbf{e}_{3}$ direction, Figure 2. Two different pressures $P_{0}$ and $P_{1}$ are applied at $X_{1}=0$ and $X_{1}=$ $L$, respectively. Locally, the fissure is limited by two parallel planes $X_{3}=$ $\pm h / 2$, with arbitrary periodicity in the $\mathbf{e}_{1}$ and $\mathbf{e}_{2}$ directions. Therefore, the $\left\langle\mathbf{v}^{* i}\right\rangle$ 's and $p^{* i}$ 's are locally dependent on the dimensionless space variable $y_{3}$, only. On an other hand, the only macroscopic dimensionless space variable present in the problem is $x_{1}$.

\subsection{DARCY'S LAW}

We first obtain as above

$$
p^{* 0}=p^{* 0}\left(x_{1}\right) \text {. }
$$
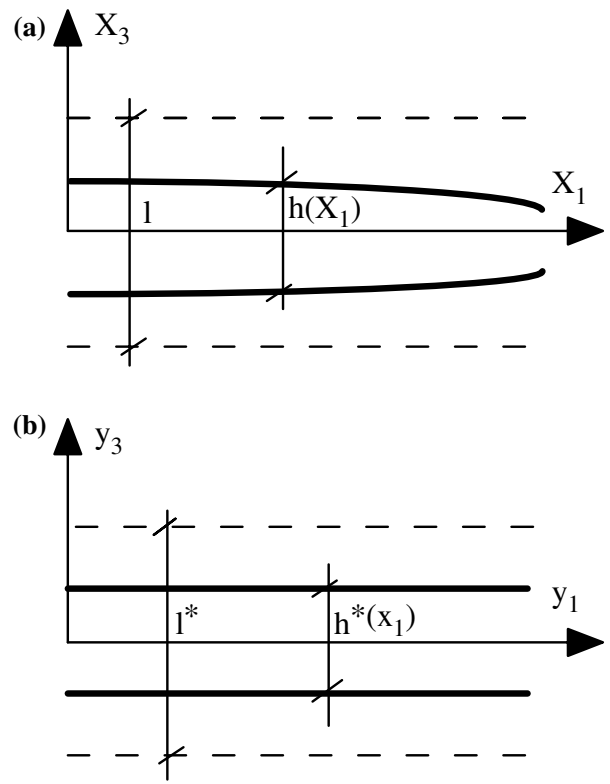

Figure 2. Plane fissure: (a) Scheme in dimensional space variable $\mathbf{X}$. (b) Scheme in dimensionless space variable $\mathbf{y}$. 
Consider now the boundary value problem for $\left\langle\mathbf{v}^{* 0}\right\rangle$ and $p^{* 1}$ on a period. The boundary value problem reduces to

$$
\begin{aligned}
& \mu^{*} \frac{\partial^{2} v_{1}^{* 0}}{\partial y_{3}^{2}}=\frac{\mathrm{d} p^{* 0}}{\mathrm{~d} x_{1}}, \quad \mu^{*} \frac{\partial^{2} v_{2}^{* 0}}{\partial y_{3}^{2}}=0, \quad \mu^{*} \frac{\partial^{2} v_{3}^{* 0}}{\partial y_{3}^{2}}=\frac{\partial p^{* 1}}{\partial y_{3}}, \\
& \frac{\partial v_{3}^{* 0}}{\partial y_{3}}=0, \quad v_{i}^{* 0}\left( \pm \frac{h}{2}\right)=0 .
\end{aligned}
$$

The solution is in the form

$$
\begin{aligned}
& v_{1}^{* 0}=-\left(\frac{h^{* 2}}{8}-\frac{y_{3}^{2}}{2}\right) \frac{\mathrm{d} p^{* 0}}{\mu^{*} \mathrm{~d} x_{1}}, \quad v_{2}^{* 0}=v_{3}^{* 0}=0, \\
& p^{* 1}=p^{* 1}\left(x_{1}\right), \\
& k_{11}^{*}=\frac{h^{* 2}}{8}-\frac{y_{3}^{2}}{2}, \quad k_{21}^{*}=k_{31}^{*}=0 \quad a_{1}^{*}=0 .
\end{aligned}
$$

Averaging over the period the volume balance of $\mathbf{v}^{* 1}$, see $\left(32_{4}\right)$ below, yields

$$
\frac{\mathrm{d}\left\langle v_{1}^{* 0}\right\rangle}{\mathrm{d} x_{1}}=0, \quad\left\langle v_{1}^{* 0}\right\rangle=-\frac{K_{11}^{*}}{\mu^{*}} \frac{\mathrm{d}\left\langle p^{* 0}\right\rangle}{\mathrm{d} x_{1}}, \quad K_{11}^{*}=\frac{h^{* 3}}{12 l^{*}} .
$$

It is possible to show that $k_{22}^{*}=k_{11}^{*}, K_{22}^{*}=K_{11}^{*}$ and the other components of $\mathbf{k}^{*}$ and $\mathbf{K}^{*}$ are zero valued.

\subsection{FIRST CORRECTOR}

By using the above expressions for $k_{i j}^{*}$ and $a_{i}^{*}$, the problem for $\mathbf{v}^{* 1}$ and $p^{* 2}$ becomes

$$
\begin{aligned}
& \mu^{*} \frac{\partial^{2} v_{1}^{* 1}}{\partial y_{3}^{2}}=\frac{\mathrm{d} p^{* 1}}{\mathrm{~d} x_{1}}, \quad \mu^{*} \frac{\partial^{2} v_{2}^{* 1}}{\partial y_{3}^{2}}=0, \quad \mu^{*} \frac{\partial^{2} v_{3}^{* 1}}{\partial y_{3}^{2}}=\frac{\partial p^{* 2}}{\partial y_{3}}, \\
& \frac{\partial v_{3}^{* 1}}{\partial y_{3}}=\frac{\partial}{\partial x_{1}}\left(\frac{k_{11}^{*}}{\mu^{*}} \frac{\mathrm{d} p^{* 0}}{\mathrm{~d} x_{1}}\right), \quad v_{i}^{* 1}\left( \pm \frac{h^{*}}{2}\right)=0
\end{aligned}
$$

which solution $\mathbf{v}^{* 1}$ is given by

$$
\begin{aligned}
& v_{1}^{* 1}=-\left(\frac{h^{* 2}}{8}-\frac{y_{3}^{2}}{2}\right) \frac{\mathrm{d} p^{* 1}}{\mu^{*} \mathrm{~d} x_{1}}, \quad v_{2}^{* 1}=0, \\
& v_{3}^{* 1}=\frac{1}{\mu^{*}} \frac{\partial}{\partial x_{1}}\left(\left(\frac{h^{* 2} y_{3}}{8}-\frac{y_{3}^{3}}{6}+\frac{h^{* 3}}{24}\right) \frac{\mathrm{d} p^{* 0}}{\mathrm{~d} x_{1}}\right),
\end{aligned}
$$


and with (59)

$$
\begin{aligned}
& v_{3}^{* 1}=\frac{1}{\mu^{*}} \frac{\partial}{\partial x_{1}}\left(\left(\frac{h^{* 2} y_{3}}{8}-\frac{y_{3}^{3}}{6}\right) \frac{\mathrm{d} p^{* 0}}{\mathrm{~d} x_{1}}\right), \\
& p^{* 2}=\left(\frac{h^{* 2}}{24}-\frac{y_{3}^{2}}{2}\right) \frac{\mathrm{d} p^{* 0}}{\mathrm{~d} x_{1}}+\bar{p}^{* 2}\left(x_{1}\right),
\end{aligned}
$$

where we have considered that $\mathbf{b}^{*}$ and $\mathbf{d}^{*}$ are of zero volume average. By averaging we have

$$
\frac{\mathrm{d}\left\langle v_{1}^{* 1}\right\rangle}{\mathrm{d} x_{1}}=0, \quad\left\langle v_{1}^{* 1}\right\rangle=-\frac{K_{11}^{*}}{\mu^{*}} \frac{\mathrm{d} p^{* 1}}{\mathrm{~d} x_{1}}, \quad\left\langle v_{2}^{* 1}\right\rangle=\left\langle v_{3}^{* 1}\right\rangle=0 .
$$

Therefore we have $\mathbf{L}^{*}=0$ and $\mathbf{N}^{*}=0$. This latter result can be also obtained from (82). On the other hand, it is possible to show that $\left\langle\mathbf{v}^{* 1}\right\rangle_{\Sigma}=\left\langle\mathbf{v}^{* 1}\right\rangle$. To the second order of approximation, the problem under consideration is described in dimensional form by

$$
\begin{aligned}
& \left\langle v_{1}\right\rangle=-\frac{K_{11}^{e f f}}{\mu} \frac{\mathrm{d} p}{\mathrm{~d} X_{1}}, \quad K_{11}^{e f f}=\frac{h^{3}}{12 l}, \\
& \frac{\partial}{\partial X_{1}}\left(\frac{K_{11}^{e f f}}{\mu} \frac{\mathrm{d} p}{\mathrm{~d} X_{1}}\right)=\mathcal{O}\left(\varepsilon^{2} \frac{\partial}{\partial X_{1}}\left(\frac{K_{11}^{e f f}}{\mu} \frac{\mathrm{d} p}{\mathrm{~d} X_{1}}\right)\right),
\end{aligned}
$$

which first relation stands for the classical Darcy's law. Due to the peculiar pore geometry under consideration, the first corrector does not account for the macroscopic heterogeneity of the medium. To point out its influence, we investigate the second corrector.

\subsection{SECOND CORRECTOR}

The third order approximation $\mathbf{v}^{* 2}$ of the velocity and the fourth order approximation $p^{* 3}$ of the pressure are determined by the following set

$$
\begin{aligned}
& \mu^{*} \frac{\partial^{2} v_{i}^{* 2}}{\partial y_{j} \partial y_{j}}+\mu^{*} \frac{\partial^{2} v_{i}^{* 0}}{\partial x_{j} \partial x_{j}}+2 \mu^{*} \frac{\partial^{2} v_{i}^{* 1}}{\partial y_{j} \partial x_{j}}-\frac{\partial p^{* 2}}{\partial x_{i}}-\frac{\partial p^{* 3}}{\partial y_{i}}=0 \text { in } \Omega_{p}^{*}, \\
& \frac{\partial v_{i}^{* 2}}{\partial y_{i}}+\frac{\partial v_{i}^{* 1}}{\partial x_{i}}=0 \text { in } \Omega_{p}^{*}, \\
& v_{i}^{* 2}=0 \text { on } \Gamma^{*}
\end{aligned}
$$

where $\mathbf{v}^{* 2}$ and $p^{* 3}$ are $\Omega$-periodic. 
In the present case, the above boundary value problem simplifies to

$$
\begin{aligned}
& \mu^{*} \frac{\partial^{2} v_{1}^{* 2}}{\partial y_{3}^{2}}-\frac{\partial^{2}}{\partial x_{1}^{2}}\left(\left(\frac{h^{* 2}}{6}-y_{3}^{2}\right) \frac{\mathrm{d} p^{* 0}}{\mathrm{~d} x_{1}}\right)-\frac{\mathrm{d} \bar{p}^{* 2}}{\mathrm{~d} x_{1}}=0, \\
& \mu^{*} \frac{\partial^{2} v_{2}^{* 2}}{\partial y_{3}^{2}}=0, \quad \mu^{*} \frac{\partial^{2} v_{3}^{* 2}}{\partial y_{3}^{2}}-\frac{\partial p^{* 3}}{\partial y_{3}}=0, \\
& \mu^{*} \frac{\partial v_{3}^{* 2}}{\partial y_{3}}-\frac{\partial}{\partial x_{1}}\left(\left(\frac{h^{* 2}}{8}-\frac{y_{3}^{2}}{2}\right) \frac{\mathrm{d} p^{* 1}}{\mathrm{~d} x_{1}}\right)=0, \quad v_{i}^{* 2}\left( \pm \frac{h^{*}}{2}\right)=0 .
\end{aligned}
$$

We are interested by $v_{1}^{* 2}$

$$
v_{1}^{* 2}=\frac{1}{\mu^{*}}\left(\frac{y_{3}^{2}}{2}-\frac{h^{* 2}}{8}\right) \frac{\mathrm{d} \bar{p}^{* 2}}{\mathrm{~d} x_{1}}+\frac{\partial^{2}}{\partial x_{1}^{2}}\left(\left(\frac{h^{* 2} y_{3}^{2}}{8}-\frac{y_{3}^{4}}{12}-\frac{5 h^{* 4}}{16 * 12}\right) \frac{\mathrm{d} p^{* 0}}{\mathrm{~d} x_{1}}\right) .
$$

By averaging the volume balance of $\mathbf{v}^{3}$, we obtain

$$
\begin{aligned}
\left\langle v_{1}^{* 2}\right\rangle= & -\frac{\mathrm{d}^{2}}{\mathrm{~d} x_{1}^{2}}\left(\frac{h^{* 2}}{8}\right) \frac{K_{11}^{*}}{\mu^{*}} \frac{\mathrm{d} p^{* 0}}{\mathrm{~d} x_{1}}-\frac{\mathrm{d}}{\mathrm{d} x_{1}}\left(\frac{7 h^{* 2}}{24}\right) \frac{K_{11}^{*}}{\mu^{*}} \frac{\mathrm{d}^{2} p^{* 0}}{\mathrm{~d} x_{1}^{2}}, \\
& -\frac{7 h^{* 5}}{720 \mu^{*} l^{*}} \frac{\mathrm{d}^{3} p^{* 0}}{\mathrm{~d} x_{1}^{3}}-\frac{K_{11}^{*}}{\mu^{*}} \frac{\mathrm{d} \bar{p}^{* 2}}{\mathrm{~d} x_{1}} \\
\left\langle v_{2}^{* 2}\right\rangle= & \left\langle v_{3}^{* 2}\right\rangle=0, \quad \frac{\mathrm{d}\left\langle v_{1}^{* 2}\right\rangle}{\mathrm{d} x_{1}}=0 .
\end{aligned}
$$

Therefore we obtain

$$
M_{11}^{*}=\frac{\mathrm{d}^{2}}{\mathrm{~d} x_{1}^{2}}\left(\frac{h^{* 2}}{8}\right) K_{11}^{*}, \quad O_{111}^{*}=\frac{\mathrm{d}}{\mathrm{d} x_{1}}\left(\frac{7 h^{* 2}}{24}\right) K_{11}^{*}, \quad P_{1111}^{*}=\frac{7 h^{* 5}}{720 l^{*}}
$$

Brinkman's coefficient $P_{1111}^{*}$ can be also obtained from (89). It is possible to show that $\left\langle\mathbf{v}^{* 2}\right\rangle_{\Sigma}=\left\langle\mathbf{v}^{* 2}\right\rangle$. In practice, we solve successively problems (59), (63) and (71) for $p^{* 0}, p^{* 1}$ and $\bar{p}^{* 2}$, respectively, with appropriate macroscopic boundary conditions.

Let us consider the corrected macroscopic behaviour. We introduce

$$
\begin{aligned}
\left\langle v_{1}^{*}\right\rangle & =\left\langle v_{1}^{* 0}\right\rangle+\varepsilon\left\langle v_{1}^{* 1}\right\rangle+\varepsilon^{2}\left\langle v_{1}^{* 2}\right\rangle+\mathcal{O}\left(\varepsilon^{3}\right), \\
\left\langle p^{*}\right\rangle & =p^{* 0}+\varepsilon p^{* 1}+\varepsilon^{2} \bar{p}^{* 2}+\mathcal{O}\left(\varepsilon^{3}\right) .
\end{aligned}
$$


The flow in direction $x_{1}$ is described by

$$
\begin{aligned}
\left\langle v_{1}^{*}\right\rangle= & -\frac{K_{11}^{*}}{\mu^{*}}\left(1+\varepsilon^{2} \frac{\mathrm{d}^{2}}{\mathrm{~d} x_{1}^{2}}\left(\frac{h^{* 2}}{8}\right)\right) \frac{\mathrm{d}\left\langle p^{*}\right\rangle}{\mathrm{d} x_{1}}-\varepsilon^{2} \frac{K_{11}^{*}}{\mu^{*}} \frac{\mathrm{d}}{\mathrm{d} x_{1}}\left(\frac{7 h^{* 2}}{24}\right) \frac{\mathrm{d}^{2}\left\langle p^{*}\right\rangle}{\mathrm{d} x_{1}^{2}} \\
& -\varepsilon^{2} \frac{7 h^{* 5}}{720 \mu^{*} l^{*}} \frac{\mathrm{d}^{3}\left\langle p^{*}\right\rangle}{\mathrm{d} x_{1}^{3}}+\mathcal{O}\left(\varepsilon^{3}\right), \\
\frac{\mathrm{d}\left\langle v_{1}^{*}\right\rangle}{\mathrm{d} x_{1}}= & \mathcal{O}\left(\varepsilon^{3}\right) .
\end{aligned}
$$

\section{Conclusion}

Flow law in porous media was studied by upscaling the pore scale description by using the method of asymptotic expansions. This method enables us to introduce correctors to Darcy's law which become nonnegligible in case of poor separation of scales. The two first correctors were investigated. Two main results are obtained. Firstly, the first corrector cancels out in the case of a macroscopically homogeneous porous medium submitted to a strong gradient of pressure. That points out the robustness of Darcy's law for such frequently encountered problems. Secondly, the second corrector introduces a Brinkman term. That shows that the Brinkman correction to Darcy's law is of relative order $\mathcal{O}\left(\varepsilon^{2}\right)$. This result deserves to be compared with Levy's investigation of Brinkman's law (Levy, 1983).

\section{Appendix A. Tensor $\mathbf{N}$}

TENSOR $\mathbf{N}^{e f f}$ VERIFIES $N_{i j k}^{e f f}=N_{i k j}^{e f f}$

We have

$$
N_{i j k}^{e f f} \frac{\partial^{2} p}{\partial X_{j} \partial X_{k}}=N_{i j k}^{e f f} \frac{\partial^{2} p}{\partial X_{k} \partial X_{j}}=N_{i k j}^{e f f} \frac{\partial^{2} p}{\partial X_{j} \partial X_{k}},
$$

which is true whatever $\frac{\partial^{2} p}{\partial X_{j} \partial X_{k}}$. Therefore, tensor $\mathbf{N}^{e f f}$ verifies

$$
N_{i j k}^{e f f}=N_{i k j}^{e f f}
$$

TENSOR $\mathbf{N}^{e f f}$ REQUIRES THAT $N_{i j k}^{e f f}=-N_{j i k}^{e f f}$ 
The periodic tensor field $k_{i m}^{*}$ is the solution of the following boundary value problem

$$
\begin{aligned}
& \frac{\partial^{2} k_{i m}^{*}}{\partial y_{j} \partial y_{j}}-\frac{\partial a_{m}^{*}}{\partial y_{i}}+I_{i m}=0, \\
& \frac{\partial k_{i m}^{*}}{\partial y_{i}}=0, \quad\left\langle\mathbf{a}^{*}\right\rangle=0 \text { in } \Omega_{p}^{*}, \\
& k_{i m}^{*}=0 \text { on } \Gamma^{*},
\end{aligned}
$$

and the periodic tensor field $n_{i k l}^{*}$ is the solution of the following boundary value problem

$$
\begin{aligned}
& \frac{\partial^{2} n_{i k l}^{*}}{\partial y_{j} \partial y_{j}}-\frac{\partial d_{k l}^{*}}{\partial y_{i}}+2 \frac{\partial k_{i l}^{*}}{\partial y_{k}}-a_{k}^{*} I_{i l}=0, \\
& \frac{\partial n_{i k l}^{*}}{\partial y_{i}}+k_{k l}^{*}-\phi^{-1} K_{k l}^{*}=0, \quad\left\langle\mathbf{d}^{*}\right\rangle=0 \text { in } \Omega_{p}^{*}, \\
& n_{i k l}^{*}=0 \text { on } \Gamma^{*},
\end{aligned}
$$

Multiply (74) by $n_{i k l}^{*}$ and integrate over $\Omega_{p}^{*}$. We have successively for the different terms, where we apply integration by parts, the divergence theorem and make use of (75), (76), (78) and (79)

$$
\begin{aligned}
\int_{\Omega_{p}^{*}} \frac{\partial^{2} k_{i m}^{*}}{\partial y_{j} \partial y_{j}} n_{i k l}^{*} \mathrm{dV}^{*} & =\int_{\Omega_{p}^{*}} \frac{\partial}{\partial y_{j}}\left(\frac{\partial k_{i m}^{*}}{\partial y_{j}} n_{i k l}^{*}\right) \mathrm{dV}^{*}-\int_{\Omega_{p}^{*}} \frac{\partial k_{i m}^{*}}{\partial y_{j}} \frac{\partial n_{i k l}^{*}}{\partial y_{j}} \mathrm{dV}^{*} \\
& =\int_{\delta \Omega_{p}^{*}}\left(\frac{\partial k_{i m}^{*}}{\partial y_{j}} n_{i k l}^{*}\right) N_{j} \mathrm{~d} \mathrm{~S}^{*}-\int_{\Omega_{p}^{*}} \frac{\partial k_{i m}^{*}}{\partial y_{j}} \frac{\partial n_{i k l}^{*}}{\partial y_{j}} \mathrm{dV}^{*} \\
& =-\int_{\Omega_{p}^{*}} \frac{\partial k_{i m}^{*}}{\partial y_{j}} \frac{\partial n_{i k l}^{*}}{\partial y_{j}} \mathrm{dV}^{*}, \\
\int_{\Omega_{p}^{*}} \frac{\partial a_{m}^{*}}{\partial y_{i}} n_{i k l}^{*} \mathrm{dV}^{*} & =\int_{\Omega_{p}^{*}} \frac{\partial\left(a_{m}^{*} n_{i k l}^{*}\right)}{\partial y_{i}} \mathrm{dV}^{*}-\int_{\Omega_{p}^{*}} \frac{\partial n_{i k l}^{*}}{\partial y_{i}} a_{m}^{*} \mathrm{dV}^{*} \\
& =\int_{\delta \Omega_{p}^{*}} a_{m}^{*} n_{i k l}^{*} N_{i} \mathrm{~d} \mathrm{~S}^{*}+\int_{\Omega_{p}^{*}} a_{m}^{*}\left(k_{k l}^{*}-\phi^{-1} K_{k l}^{*}\right) \mathrm{dV}^{*} \\
& =\int_{\Omega_{p}^{*}} a_{m}^{*} k_{k l}^{*} \mathrm{dV}^{*}, \\
\int_{\Omega_{p}^{*}} n_{i k l}^{*} I_{i m} \mathrm{dV}^{*} & =\int_{\Omega_{p}^{*}} n_{m k l}^{*} \mathrm{dV}^{*}=\Omega^{*} N_{m k l}^{*},
\end{aligned}
$$

that yields by regrouping all terms

$$
\Omega^{*} N_{m k l}^{*}=\int_{\Omega_{p}^{*}} \frac{\partial k_{i m}^{*}}{\partial y_{j}} \frac{\partial n_{i k l}^{*}}{\partial y_{j}} \mathrm{dV}^{*}+\int_{\Omega_{p}^{*}} a_{m}^{*} k_{k l}^{*} \mathrm{dV}^{*}
$$


Multiply now (77) by $k_{i m}^{*}$ and integrate over $\Omega_{p}^{*}$. By following the same route, we obtain

$$
\int_{\Omega_{p}^{*}} \frac{\partial k_{i m}^{*}}{\partial y_{j}} \frac{\partial n_{i k l}^{*}}{\partial y_{j}} \mathrm{dV}^{*}=2 \int_{\Omega_{p}^{*}} \frac{\partial k_{i l}^{*}}{\partial y_{k}} k_{i m}^{*} \mathrm{dV}^{*}-\int_{\Omega_{p}^{*}} a_{l}^{*} k_{k m}^{*} \mathrm{dV}^{*} .
$$

The two last relations yields tensor $\mathbf{N}^{*}$ in the form

$$
\Omega^{*} N_{m k l}^{*}=2 \int_{\Omega_{p}^{*}} \frac{\partial k_{i l}^{*}}{\partial y_{k}} k_{i m}^{*} \mathrm{dV}^{*}-\int_{\Omega_{p}^{*}} a_{l}^{*} k_{k m}^{*} \mathrm{dV}^{*}+\int_{\Omega_{p}^{*}} a_{m}^{*} k_{k l}^{*} \mathrm{dV}^{*} .
$$

Note that tensor $\mathbf{N}^{*}$ can be determined from tensor fields $\mathbf{k}^{*}$ and $\mathbf{a}^{*}$. We use expression (82) to demonstrate the antisymmetry of tensor $\mathbf{N}^{*}$. With the symmetry property (73) applied successively to $N_{m k l}^{*}$ and $N_{k m l}^{*}$, we have

$$
\begin{aligned}
& \Omega^{*} N_{m k l}^{*}=2 \int_{\Omega_{p}^{*}} \frac{\partial k_{i k}^{*}}{\partial y_{l}} k_{i m}^{*} \mathrm{dV}^{*}-\int_{\Omega_{p}^{*}} a_{k}^{*} k_{l m}^{*} \mathrm{dV}^{*}+\int_{\Omega_{p}^{*}} a_{m}^{*} k_{l k}^{*} \mathrm{dV}^{*}, \\
& \Omega^{*} N_{k m l}^{*}=2 \int_{\Omega_{p}^{*}} \frac{\partial k_{i m}^{*}}{\partial y_{l}} k_{i k}^{*} \mathrm{dV}^{*}-\int_{\Omega_{p}^{*}} a_{m}^{*} k_{l k}^{*} \mathrm{dV}^{*}+\int_{\Omega_{p}^{*}} a_{k}^{*} k_{l m}^{*} \mathrm{dV}^{*} .
\end{aligned}
$$

After adding term by term the two last equalities and using relation (76), we finally obtain

$$
\begin{aligned}
\Omega^{*}\left(N_{m k l}^{*}+N_{k m l}^{*}\right) & =2 \int_{\Omega_{p}^{*}}\left(\frac{\partial k_{i k}^{*}}{\partial y_{l}} k_{i m}^{*}+\frac{\partial k_{i m}^{*}}{\partial y_{l}} k_{i k}^{*}\right) \mathrm{dV}^{*} \\
& =2 \int_{\Omega_{p}^{*}} \frac{\partial\left(k_{i k}^{*} k_{i m}^{*}\right)}{\partial y_{l}} \mathrm{dV}^{*}=2 \int_{\delta \Omega_{p}^{*}} k_{i k}^{*} k_{i m}^{*} N_{l} \mathrm{dS}^{*}=0 .
\end{aligned}
$$

\section{Appendix B: Tensor $\mathbf{P}$}

The periodic tensor field $p_{i k l m}^{*}$ is the solution of the following boundary value problem

$$
\begin{aligned}
& \frac{\partial^{2} p_{i k l m}^{*}}{\partial y_{j} \partial y_{j}}+k_{i m}^{*} I_{k l}-\frac{\partial f_{k l m}^{*}}{\partial y_{i}}+2 \frac{\partial n_{i k l}^{*}}{\partial y_{m}}-d_{l m}^{*} I_{i k}=0, \\
& \frac{\partial p_{i k l m}^{*}}{\partial y_{i}}+n_{m k l}^{*}-\phi^{-1} N_{m k l}^{*}=0 \text { in } \Omega_{p}^{*}, \\
& p_{i k l m}^{*}=0 \text { on } \Gamma^{*} .
\end{aligned}
$$

Multiply (74) written for $k_{i n}^{*}$ by $p_{i k l m}^{*}$ and integrate over $\Omega_{p}^{*}$. We have successively for the different terms, where we apply integration by parts, the 
divergence theorem and make use of (75), (76), (85) and (86)

$$
\begin{aligned}
\int_{\Omega_{p}^{*}} \frac{\partial^{2} k_{i n}^{*}}{\partial y_{j} \partial y_{j}} p_{i k l m}^{*} \mathrm{dV}^{*} & =\int_{\Omega_{p}^{*}} \frac{\partial}{\partial y_{j}}\left(\frac{\partial k_{i n}^{*}}{\partial y_{j}} p_{i k l m}^{*}\right) \mathrm{dV}^{*}-\int_{\Omega_{p}^{*}} \frac{\partial k_{i n}^{*}}{\partial y_{j}} \frac{\partial p_{i k l m}^{*}}{\partial y_{j}} \mathrm{dV}^{*} \\
& =\int_{\delta \Omega_{p}^{*}}\left(\frac{\partial k_{i n}^{*}}{\partial y_{j}} p_{i k l m}^{*}\right) N_{j} \mathrm{dS}^{*}-\int_{\Omega_{p}^{*}} \frac{\partial k_{i n}^{*}}{\partial y_{j}} \frac{\partial p_{i k l m}^{*}}{\partial y_{j}} \mathrm{dV}^{*} \\
& =-\int_{\Omega_{p}^{*}} \frac{\partial k_{i n}^{*}}{\partial y_{j}} \frac{\partial p_{i k l m}^{*}}{\partial y_{j}} \mathrm{dV}^{*}, \\
\int_{\Omega_{p}^{*}} \frac{\partial a_{n}^{*}}{\partial y_{i}} p_{i k l m}^{*} \mathrm{dV}^{*} & =\int_{\Omega_{p}^{*}} \frac{\partial\left(a_{n}^{*} p_{i k l m}^{*}\right)}{\partial y_{i}} \mathrm{dV}^{*}-\int_{\Omega_{p}^{*}} \frac{\partial p_{i k l m}^{*}}{\partial y_{i}} a_{n}^{*} \mathrm{dV}^{*} \\
& =\int_{\delta \Omega_{p}^{*}} a_{n}^{*} p_{i k l m}^{*} N_{i} \mathrm{~d} \mathrm{~S}^{*}+\int_{\Omega_{p}^{*}} a_{n}^{*}\left(n_{m k l}^{*}-\phi^{-1} N_{m k l}^{*}\right) \mathrm{d} \mathrm{V}^{*} \\
& =\int_{\Omega_{p}^{*}} a_{n}^{*} n_{m k l}^{*} \mathrm{dV}^{*}, \\
\int_{\Omega_{p}^{*}} p_{i k l m}^{*} I_{i n} \mathrm{dV}^{*} & =\int_{\Omega_{p}^{*}} p_{n k l m}^{*} \mathrm{dV}^{*}=\Omega^{*} P_{n k l m}^{*},
\end{aligned}
$$

that yields by regrouping all terms

$$
\Omega^{*} P_{m k l n}^{*}=\int_{\Omega_{p}^{*}} \frac{\partial k_{i n}^{*}}{\partial y_{j}} \frac{\partial p_{i k l m}^{*}}{\partial y_{j}} \mathrm{dV}^{*}+\int_{\Omega_{p}^{*}} a_{n}^{*} n_{m k l}^{*} \mathrm{dV}^{*}
$$

Multiply now (84) by $k_{i n}^{*}$ and integrate over $\Omega_{p}^{*}$. By following the same route, we obtain

$$
\begin{aligned}
\int_{\Omega_{p}^{*}} \frac{\partial k_{i n}^{*}}{\partial y_{j}} \frac{\partial p_{i k l m}^{*}}{\partial y_{j}} \mathrm{dV}^{*}= & 2 \int_{\Omega_{p}^{*}} \frac{\partial n_{i k l}^{*}}{\partial y_{m}} k_{i n}^{*} \mathrm{dV}^{*}-\int_{\Omega_{p}^{*}} d_{l m}^{*} k_{n k}^{*} \mathrm{dV}^{*} \\
& +\int_{\Omega_{p}^{*}} k_{i m}^{*} k_{i n}^{*} I_{k l} \mathrm{dV}^{*}
\end{aligned}
$$

The two last relations yields tensor $\mathbf{P}^{*}$ in the form

$$
\begin{aligned}
\Omega^{*} P_{n k l m}^{*}= & 2 \int_{\Omega_{p}^{*}} \frac{\partial n_{i k l}^{*}}{\partial y_{m}} k_{i n}^{*} \mathrm{dV}^{*}-\int_{\Omega_{p}^{*}} d_{l m}^{*} k_{n k}^{*} \mathrm{dV}^{*}+\int_{\Omega_{p}^{*}} a_{n}^{*} n_{m k l}^{*} \mathrm{dV}^{*} \\
& +\int_{\Omega_{p}^{*}} k_{i m}^{*} k_{i n}^{*} I_{k l} \mathrm{dV}^{*}
\end{aligned}
$$

Note that tensor $\mathbf{P}^{*}$ can be determined from tensor fields $\mathbf{k}^{*}, \mathbf{a}^{*}, \mathbf{n}^{*}$ and $\mathbf{d}^{*}$. 


\section{References}

Auriault, J.-L.: 1991, Heterogeneous medium. Is an equivalent macroscopic description possible?, Int. J. Engng Sci. 29(7), 785-795.

Auriault, J.-L. and Adler, P.: 1995, Taylor dispersion in porous media: analysis by multiple scale expansions, Adv. Water Res. 18(4), 217-226.

Bensoussan, A., Lions, J.-L. and Papanicolaou, G.: 1978, Asymptotic Analysis for Periodic Structures, North Holland.

Ene H. I. and Sanchez-Palencia, E.: 1975, Equations et phénomènes de surface pour l'écoulement dans un modèle de milieux poreux, Journal de Mécanique 14(1), 73-108.

Goyeau, B., Benihaddadene, T., Gobin, D. and Quintard, M.: 1997, Averaged momentum equation for flow through a nonhomogeneous porous structure, Transp. Porous Media 28, 19-50.

Goyeau, B., Benihaddadene, T., Gobin, D. and Quintard, M.: 1999, Numerical calculation of the permeability in a dentritic mushy zone, Metall. Mater. Trans. B 30B, 613-622.

Levy, T.: 1983, Fluid flow through an array of fixed particles, Int. J. Engng. Sci. 21(1), 11-23.

Murdoch A. I. and Hassanizadeh S. M.: 2002, Macroscale balance relations for bulk, interfacial and common line systems in multiphase flows through porous media on the basis of molecular considerations, Int. J. Multiphase Flow 28, 1091-1123.

Sanchez-Palencia, E.: 1980, Non Homogeneous Media and Vibration Theory, Lecture notes in Physics 127, Springer. 